\title{
25 Research Soure \\ Effects of Enriched Environment on Microglia and Functional White Matter Recovery in Rats with Post stroke Cognitive Impairment
}

\section{Yi-Sha Guo}

Shanghai University of Sport

\section{Mei Yuan}

Shanghai University of Sport

\section{Yu Han}

Shanghai University of Sport

\section{Xin-Ya Shen}

Shanghai University of Traditional Chinese Medicine

\section{Zhen-Kun Gao}

Shanghai University of TCM: Shanghai University of Traditional Chinese Medicine

Xia Bi ( $\square$ bixiash@163.com )

Shanghai Pudong New District Zhoupu Hospital https://orcid.org/0000-0002-4834-6954

\section{Research}

Keywords: MCAO, ischemic stroke, enriched environment, white matter, microglia, inflammation

Posted Date: May 5th, 2021

DOI: https://doi.org/10.21203/rs.3.rs-450389/v1

License: (c) (1) This work is licensed under a Creative Commons Attribution 4.0 International License.

Read Full License

Version of Record: A version of this preprint was published at Neurochemistry International on February 1st, 2022. See the published version at https://doi.org/10.1016/j.neuint.2022.105295. 


\section{Abstract}

Background: White matter damage is an important contributor to cognitive impairment after stroke. This study was designed to explore the beneficial effects of enriched environment (EE) on white matter recovery and cognitive dysfunction after stroke, and further explore the potential mechanism of EE on white matter recovery from the perspective of microglia and microglia-mediated neuroinflammation.

Methods: Male SD rats underwent middle cerebral artery occlusion(MCAO) or sham surgery. During the MCAO operation, a laser Doppler blood flow meter was used to monitor the blood flow to ensure the success of the model. At 72 hours after the operation, 3 rats were selected for TTC staining to identify the infarct size. One week after surgery, the rats were randomly assigned into four different groupsMCAO+standard environment (SE), MCAO+enriched environment(EE), Sham+SE and Sham+EE for 4 weeks. At four weeks after MCAO surgery, neurological function deficiency condition and cognitive function were assessed using Longa score and Morris Water Maze prior to euthanasia. The loss or regeneration of myelin was stained with LFB, the expression of myelin regeneration-related protein and microglia protein was quantified by western blot and immunofluorescence, and the level of inflammatory factors was measured by ELISA.

Results: EE treatment remarkably decreased the neurological deficit score, ameliorated the cognitive functional deficit in MCAO rats. Furthermore, EE alleviated white matter lesions and demyelination, increased myelin basic protein expression and decreased the number of activated microglia in the hippocampus of MCAO rats. In addition, ELISA analysis indicated that EE decreased the level of IL-1 $\beta$, IL-6, which further suggests that EE may reduce the level of pro-inflammatory factors by affecting the expression of microglia, provide a benefit physiological environment for myelin recovery, and improve post stroke cognitive impairment.

Conclusions: Our results suggest that exposure to EE substantially reduced microglia damage/activation , decreased the level of pro-inflammatory cytokins induced by microglia, protected and promote white matter recovery to improve cognitive function in injured area after stroke. Our findings also indicate exposure to EE is beneficial for patients with white matter impairment characterised by white matter disease-related inflammation.

\section{Introduction}

Patients with stroke often suffer from cognitive impairment after stroke. The prevalence of post-stroke cognitive impairment is between $20 \%$ and $80 \%(1)$. The underlying mechanism of cognitive impairment after stroke is unclear. According to previous studies, hippocampus and white matter lesions(WMLs) is one of the important pathogenesis of post-stroke cognitive impairment. White matter lesions are common imaging manifestations of subclinical ischemic brain parenchymal damage caused by cerebellar vascular disease(2). Studies have confirmed that white matter lesions and lacunar infarction impair psychomotor speed, executive function and overall cognitive function and so on(3). However, there's no 
unequivocally efficacious treatment and mechanisms for promoting white matter recovery after stroke are still unclear.

Previous studies have showed that neuroinflammation plays a key role in white matter injury and cognitive dysfunction induced by cerebral ischemia-reperfusion $(4,5)$. Neuroinflammation affects the demyelination and remyelination of oligodendrocytes. Glial cells are the primary components of the periinfarct environment in the central nervous system (CNS) and are related to immune regulation after stroke(5). Microglia are the main type of glial cells, which are responsible for surveying the homeostasis of the central nervous system and regulating internal immune response. Increasing evidence suggests that microglia exert beneficial and detrimental effects during ischemic stroke. Microglia can activate rapidly and persist for several weeks after ischemic stroke, which release inflammatory factors to cause nerve tissue damage(6-8). However, alternatively activated microglia can secrete anti-inflammatory cytokines and neurotrophic factors, which is beneficial for recovery after ischemic stroke.

The enriched environment $(\mathrm{EE})$ is defined as stimulating the brain by improving the social or physical environment of an individual or animal. Compared to standard environments, animals in EE usually receive more social, sensory and cognitive stimulation(9). EE is usually characterized by rich social interaction (multiple placement), spacious living environment (large-volume cage), and diverse sensory and motor stimulation (placement of toys, running wheels and/or slides, etc.). The previous research of the research group and other related literatures have shown that EE can significantly improve the cognitive function after stroke (9). In addition, a large number of studies have shown that EE can promote white matter recovery and remyelination. Thomas A. Forbes et al. found that in perinatal brain injury, the early and continuous enriched environment can selectively enhance the endogenous repair of developing white matter by enhancing oligodendrocyte formation, myelination and functional recovery. This implies that $E E$ can accelerate the response of endogenous oligodendrocyte genes to injury and induce additional changes in genes that promote myelination(10). In experimental autoimmune encephalomyelitis (EAE) mice in a demyelinating model, the rich environment promotes the expression of oligodendrocyte markers in cells recruited from the injured area (11). Another similar finding was found in the injury area of focal cerebral ischemia. 6-month-old spontaneously hypertensive rats were ligated through the right middle cerebral artery to cause cortical infarction. After five weeks of rearing in a rich environment, NG2 positive cells (a marker of oligodendrocyte expression) in the injured area were found to increase(12). The above studies have proved that enriched environment plays a very important role in regulating the remyelination after central white matter injury, but the underlying molecular mechanism of its regulation is still unclear.

Here, we determine-for the first time-the effects of EE on injured white matter post-ischemic stroke, and provide a much-needed bridge between the myelin plasticity and WM-associated cognitive function recovery. EE intervention after ischemic stroke enhanced myelination, resulting in improved behavioral performance. Further, studies have confirmed that microglia activation and inflammation caused by cerebral ischemia affect the remyelination of damaged areas $(13,14)$, so we begin to explore microgliamediated inflammatory mechanisms underlying enrichment-induced recovery in MCAO. 


\section{Materials And Methods \\ 2.1 Animals}

Adult male Sprague Dawley rats were used in this study (6-8 weeks old, $250 \pm 20 \mathrm{~g}$, Shanghai SLRC Laboratory Animal Co., Ltd., Shanghai, China). The rats were housed in a controlled condition with a $12 \mathrm{~h}$ light/dark cycle ( 6 am- 6 pm, day; 6 pm- 6 am,night), a controlled temperature ( 20 to $24^{\circ} \mathrm{C}$ ) and humidity $(40-70 \%)$, and free access to food and water for three day to adapt to the environment. After three-days acclimatisation, all rats were randomly divided for either middle cerebral artery occlusion(MCAO) or sham surgery. The experimental protocol of this study were approved by the Animal Ethical Committee of Shanghai University of Traditional Chinese Medicine.

\subsection{Establishment of PSCI model}

All SD rats were fed adaptively for 3 days before surgery. During the operation, the rats were injected with $5 \%$ isoflurane(Art No. T8877, Sigma, USA) and supplemented with $1-1.5 \%$ isoflurane. The rectal temperature was maintained at $37.0 \pm 0.5^{\circ} \mathrm{C}$ during the operation. MCAO model was achieved by inserting a monofilament $(0.32 \pm 0.02 \mathrm{~mm}$ )(Rayward Life Technology Co., Ltd., Shenzhen, China) into the left common carotid artery of the rat via internal carotid artery. And cerebral blood flow in MCA area was monitored by laser Doppler flowmeter(Moor Instruments, Devon, USA) (Fig. 1). Effective blockade was confirmed by cerebral blood flow by $₫ 70 \%$. After 90 minutes, the monofilaments were removed, skin incisions were sutured, and the rats were put back into the cage after heat preservation. In the sham operation group, the other surgical methods were the same as above except the insertion of nylon filament. After recovering from anesthesia, neurobehavioral were scored using the Longa scoring system, and rats with scores of 0 or 4 were excluded from the study. Rats with a score of 1-3 were considered as the models of success and were placed in standard conditions. After 24 hours, the rats were randomly assigned to each group.

\subsection{EE paradigm and animal groups}

Animals were initially assigned into two main groups, sham and MCAO. Three days after the surgery, rats were randomly divided to four subgroups: (1) sham + standard environment $(\mathrm{SE})(\mathrm{n}=7) ;(2)$ sham + environment enrichment(EE) $(n=7)$; (3) MCAO + standard environment(SE) $(n=7)$ and (4) MCAO + environment environment(EE) $(n=7)$.

The home cage in EE group was $39 \mathrm{~cm}$ wide $\times 54.5 \mathrm{~cm}$ long $\times 20 \mathrm{~cm}$ high and contained a ladder, plastic pipes and tunnels, running wheels and small boxes. Meanwhile, in order to simulate the rehabilitation environment of the clinical ward, the MCAO group and the sham operation group were mixed and randomly divided into the EE group (7 rats in each enriched environment cage), because stroke patients may also come into contact with healthy people, who may give them different social stimuli. Standard environment $(15 \mathrm{~cm}$ wide $\times 29.5 \mathrm{~cm}$ long $\times 12.5 \mathrm{~cm}$ high) is normal living conditions, no objects, 3 rats per cage. 


\subsection{Quantification of infarct volume}

The rats were sacrificed 72 hours after the completion of cerebral ischemia surgery, and the fresh brain tissue was quickly frozen and cut into coronal sections of about $1 \mathrm{~mm}$. Using 2,3,5-Triphenyltetrazolium Chloride (T8877, Sigma), TTC was dehydrogenase in living tissue Reduced to red methoxymidine products and pale staining corresponding to infarct areas. The measurement of infarct volume is available. The infarcted tissue was analyzed using digital image analysis software (Sigmascan Pro, Jandel, San Rafael, CA, USA). The infarct size of each section was measured ( $1 \mathrm{~mm})$ by subtracting the total area of the contralateral hemisphere from the non-infarct area of the ipsilateral hemisphere, and then the final infarct volume was calculated by adding up the infarct area of each section and multiplying it by section thickness.

\subsection{Neurobehavioral assessment}

Neurobehavioral function tests were assessed 7, 14, 28 days after MCAO by two researchers who were blinded to the test groups. The Longa scoring system was used to detect neurological impairment: the score was 0 , and there was no neurological impairment. 1 point (full extension of right forepaw) mild deficiency; 2 points (rotated right), 3 points (totward right), moderate inadequacy; 4 points (inability to walk independently, decreased consciousness), serious deficiency.

\subsection{Morris Water Maze (MWM) Test}

The MWM test was performed 28 days after MCAO to evaluate the ability of spatial learning and memory of the rats. The test was performed as described in the protocols(15). The experiment consists of two evaluations: the space acquisition test and the detection test. All the experiments were carried out in a quiet environment. The pool is a circular pool, $122 \mathrm{~cm}$ in diameter, $50 \mathrm{~cm}$ high and $30 \mathrm{~cm}$ deep, divided almost into four equal quadrants. The water temperature is about $20 \sim 22^{\circ} \mathrm{C}$. The $10 \mathrm{~cm}^{2}$ concealed circular platform is located in the NE quadrant and submerged $0.5 \sim 1.0 \mathrm{~cm}$ below the surface of the water. The water maze test will take seven days. The first day of the test was an adaptive swim, followed by five consecutive days of spatial acquisition trials. There are four trials a day. The starting position is SE, S, NW, W, as shown in Table 1. If the mouse does not reach the platform within 90 seconds, guide the rats to the platform. After the animal reached the escape table, it was allowed to remain there for 15 seconds before another experiment was conducted. The escape latency is defined as the time it takes for the animal to reach the escape platform. The probe test was carried out on the seventh day. When the escape platform was removed from the pool, the rats could swim in the pool for 90 seconds. The time the animal stayed in the target quadrant and the distance it traveled were recorded, as well as the number of times the animal crossed the previous platform area. All swim routes were monitored using image detectors and analyzed using ANY-maze software (ANY-maze, Stoelting Co., IL, USA).

\subsection{Histological Analysis of WM Injury of hippocampal area}

The animals were deeply anesthetized with isoflurane, followed by perfusion with saline and then perfusion with $4 \%$ paraformaldehyde in 0.1-M phosphate-buffer saline (PBS; PH 7.4). The brains were 
removed and placed overnight in the same fixative at $4^{\circ} \mathrm{C}$, then stored in PBS with $15 \%$ and $30 \%$ sucrose until the tissue sank. Coronal sections (about $8 \mathrm{~mm}$ thick), including the hippocampus and cortex, were placed in an optimal cutting temperature (OCT) compound, frozen in liquid nitrogen, and then cut into $8 \mu \mathrm{m}$ sections using a microtomy-cryo thermostat. Luxol-Fast Blue (LFB) staining was used to assess the degree of demyelination or regeneration. We took 3 slices from each rat and randomly selected 3 regions of hippocampal CA3 for evaluation. Imagej is used to quantify LFB staining. The image was taken using a bright field microscope (Leitz Dialux 20, Leica) with a 5 objective lens and a Lumenera Infinity digital camera (Lumenera Corporation, Canada). The areas covered by the LFB stain were expressed as a percentage of the area of the entire field of view assessed.

\subsection{Immunofluorescence Staining}

For immunofluorescence, frozen sections of brain tissue were fixed in $4 \%$ paraformaldehyde (PFA) overnight. After fixing the brain tissue on the microtome with OTC, cut multiple sets of frozen sections ( $8 \mu \mathrm{m}$ thick, hippocampus) in a cryostat (Leica CM1950) at $24^{\circ} \mathrm{C}$, and collect every 3 microtome on each Super Frost Plus slide, then store at $80^{\circ} \mathrm{C}$ for further dyeing. In order to perform immunofluorescence staining, the brain sections were fixed with $4 \%$ paraformaldehyde for 10 minutes at room temperature (25 $\sim 26^{\circ} \mathrm{C}$ ) and blocked with $10 \%$ BSA for 60 minutes. The slides were incubated with primary antibodies of IBA-1 (1:1000, Abcam, ab178847), MBP (1:3000, Servicebio, GB12226), NG2 (1:1000, Servicebio, DF12589) at $4^{\circ} \mathrm{C}$ overnight. After rinsing 3 times with PBS, we incubate the brain slices with fluorescently coupled secondary antibody (anti-mouse, 1:3000) at room temperature for 60 minutes. Images were taken using a confocal microscope (LeicaTCS SP2). For immunofluorescence staining analysis, four fields of view (200x) of each area were photographed under a confocal microscope (Leica TCS SP2), and Image-Pro Plus 6.0 (Media Cyber Netics Inc., MD, USA) aims to evaluate the proportion of positive areas.

\subsection{Western Blot Analysis}

We collect bilateral intact hippocampal tissue samples and sonicate them in homogeneous buffer (RIPA with protease cocktail inhibitor, phosphatase inhibitor, and phenylmethanesulfonyl fluoride). Perform SDS-PAGE on an equal amount of sample on a $10 \%$ gel, and transfer the protein to a PVDF membrane (Millipore, Massachusetts, USA). Then the membrane was incubated overnight at $4^{\circ} \mathrm{C}$ with the following primary antibodies: IBA1 (1:1000, Abcam, ab178847), MBP (1:3000, Servicebio, GB12226), PDGFR-a (1:750, Servicebio, GB111342) and GAPDH (1:1000, Servicebio, DF12589). After washing 3 times with TBST, the membrane was incubated with horseradish peroxide (HRP)-conjugated secondary antibody for 60 minutes at room temperature and visualized by chemiluminescence (Servicebio, G2014). The results were recorded with an imaging system (Adobe, CA, USA). The optical density ratio of the target band to GAPDH is used as the relative expression of the target protein.

\subsection{Enzyme-linked immunosorbent assay (ELISA).}

Blood samples were taken at 28 days after MCAO induction to separate serum. An ELISA was 
performed to measure IL-1 $\beta$ (cat. no., RLB00, R\&D Systems Inc., Minneapolis, MN, USA) levels in the serum and hippocampus by measuring the optical density (OD) at $450 \mathrm{~nm}$. The experiment was repeated at least three times to calculate the average value.

\subsection{Statistical analysis}

Data are expressed as mean SD. Using IBM SPSS statistics 25 software, the t-test was used to compare the western blot immunofluorescence staining, and ELISA data in each group. The Mann-Whitney $U$ test or Kruskal-WallisH test was performed for non-parametric analysis, and repeated measures one-way analysis of variance (ANOVA) followed by Scheffe's post hoc comparison was used to evaluate the differences in Longa scores, escape latency and platform crossing time among groups. In all analyses, $\mathrm{P}$ $<0.05$ was defined as statistically significant.

Table 1

Morris water maze spatial(hidden platform)start positions

\begin{tabular}{|lllll|}
\hline \multicolumn{4}{|l|}{ Acquistion } & \multicolumn{3}{l|}{} \\
\hline Day & Trial 1 & Trial 2 & Trial 3 & Trial 4 \\
\hline 1 & S & W & NW & SE \\
\hline 2 & NW & S & SE & W \\
\hline 3 & SE & NW & W & S \\
\hline 4 & W & SE & S & NW \\
\hline 5 & S & NW & W & SE \\
\hline 6 (Probe) & SW & & & \\
\hline \multicolumn{4}{|l}{ NW, Northwest; S, south; SE, southeast; W, west. } \\
\hline
\end{tabular}

\section{Results}

\subsection{Successful establishment of cerebral ischemia- reperfusion model}

In order to ensure the successful modeling of the stroke model,, the rat's cerebral blood flow changes are monitored and recorded in real time by a laser Doppler blood flow meter when performing the stroke model operation. When the cerebral blood flow decreases by more than $70 \%($ Fig. 1B), the occlusion is successful. After the blood flow was blocked for 90 minutes, the monofilament was removed to restore the blood supply to the brain. At this time, the color of the cerebral ischemic area can be seen from dark blue to cyan, yellow and red in the picture(Fig. 1A). In addition, at 72 hours after MCAO, 3 rats were 
selected for TTC staining. The white is the infarct area. It can be seen from the Fig. 2 that there are obvious white areas in the MCAO surgery group, which proves that the model is successful.

\subsection{EE treatment ameliorated reduced neurological scores and cognitive function after cerebral ischemia-reperfusion}

We first evaluated their neurological impairment scores (Longa) at 7, 14, and 28 days after reperfusion. After EE treatment, the scores of the three stages were significantly reduced. But in the SE group, there was no statistical difference in the scores of the three stages. Based on the results of LFB staining and myelin marker protein suggesting a remyelination response after MCAO surgery, we investigated EEinduced cognitive function recovery using a Morris Water Maze (Fig. 3). This test is a useful and reproducible assessment of spatial learning and memory ability. As shown in Fig. 3, SE group performed poorly on MWM task vs. EE group regardless of MCAO or sham. The track plots showed the trace of rats in the tank during the spatial acquisition on the fifth day(Fig. 3B) and the probe test on the sixth day(Fig. 3C). The fewer traces recorded from the spatial acquisition test, the stronger spatial learning ability of rats. The paths of MCAO(SE) group were significantly more than those in the other groups(Fig. 3B). In the probe trial, the more concentrated the swimming trajectory of the rat is in the quadrant of the platform, the better the memory ability of the rat is represented. The rats of MCAO(SE) had significantly fewer track plots in the quadrant of the platform compared with the other groups. The results showed the change in escape latency from five-day test and the crossing former platform times among different groups, and the EE group spent less time finding the platform(Fig. 3B) and more number of times cross former platform(Fig. 3C) compared with the SE group regardless of MCAO or Sham. These behavioral studies strongly align with our histological findings, suggesting that EE ameliorates the effects of cerebral ischemia by increasing OL maturation and promoting myelination required for WM dependent functional improvement.

\subsection{Enriched environment promotes the regeneration of myelin sheath in cerebral ischemic areas}

To evaluate remyelination post-MCAO, we performed LFB staining on the hippocampus of Rats. In the MCAO (SE) group, there was obvious myelin sheath disappearance at the place indicated by the arrow, while the MCAO (EE) group had more myelin sheath and there is a statistical difference, but the myelin content still did not return to normal compared with the Sham group(Fig. 4). To further investigate the effects of EE under cerebral ischemic conditions, we analyzed PDGFR-a(Oligodendrocyte precursor cell Marker) and MBP(Mature oligodendrocytes Marker) expression in the hippocampus using western blot. As predicted, MBP expression from MCAO in EE led to a significant increase compared to MCAO(SE) group (Fig. 5A,B). However, there is no difference in PDGFR-abetween the four group (Fig. 5A,B). We also performed immunofluorescence double staining of oligodendrocyte precursor cells and myelin markers(NG2 and MBP, respectively), and analyzed the proportion of positive cells in the CA3 area of the hippocampus. The results showed that there was no difference in NG2(Oligodendrocyte precursor cell Marker) between the groups. Regardless of whether the MCAO model group or the sham operation group, 
the mature myelin area accounted for more EE than SE, but there was no statistical difference(Fig. 5C,D). We guessed that it might be because the sample size was too small, which led to errors in the results. However, combining the results of LFB staining, western blot and immunofluorescence, EE is beneficial to myelination, especially in the MCAO model group.

\subsection{EE reduces the expression of microglia in the ipsilateral hippocampus after cerebral ischemia-reperfusion}

Many studies have shown that microglia are involved in the white matter recovery process after ischemic stroke. Therefore, in order to explore whether EE promotes remyelination after stroke by regulating the expression level of microglia. We first analyzed the protein expression level of ipsilateral microglia by western blot and immunofluorescence(CA1, CA3), and found that compared with MCAO (SE) group, MCAO (EE) significantly reduced the protein expression level of microglia(Fig. 6B, C, D). Then, after immunofluorescence analysis of the CA3 area of the contralateral hippocampus of rats brain, we found that there was no statistical difference in the expression level of microglia between the MCAO (SE) and MCAO (EE) groups(Fig. 6E).

\subsection{EE suppresses proinflammatory cytokines levels in serum after cerebral ischemic-reperfusion}

Microglia are the main inflammatory cells in the central nervous system. Their role is related to their classification. With the activation of microglia after ischemic stroke, the phenotype of microglia has undergone some changes, which can be simply divided into pro-inflammatory and anti-inflammatory types, namely $\mathrm{M} 1$ and $\mathrm{M} 2$, respectively(16). $\mathrm{M} 1$ is a pro-inflammatory cell that secretes some proinflammatory cytokines, such as interleukin-1 beta (IL-1 $\beta$ ) and IL-6 were expressed by microglia following ischemic stroke, which are primarily neurotoxic(8). Type $\mathrm{M} 2$ is related to anti-inflammatory and secretes some anti-inflammatory cytokines(17). EE reduces the activation of microglia. We speculate that EE inhibits the M1 typing of microglia at this time, thereby reducing the release of pro-inflammatory cytokines. Therefore, we extracted the serum of rats 28 days after MCAO and performed ELISA analysis, and found that the levels of representative pro-inflammatory cytokines IL-1 ßand IL- 6 were lower than those of MCAO (SE) in the MCAO (EE) group(Fig. 7).

\subsection{Correlations Analysis}

The Pearson's correlation coefficients test showed that there were significant negative correlations between the cognitive function outcomes and MBP expression levels $(r=-0.0196, p \otimes 0.0001$, Fig. $5 A)$ and significant positive correlations between the cognitive function outcomes and BDNF expression levels ( $r$ $=0.8790, p=0.0002$, Fig. 5B) at 28 days after MCAO. Our data indicate that increase of MBP expression and decrease of microglia are correlated with improved functional recovery of stroke animals.

\section{Discussion}

Our research confirmed the effect of EE on the recovery of white matter after stroke and its cognitive function, and further explored the microglia-mediated neuroinflammation mechanism that EE promotes 
the recovery of white matter post-stroke. The results of the study found that EE can promote the recovery of white matter damage caused by stroke, is conducive to remyelination, and improves the cognitive function of MCAO rats. In addition, the results of the study also found that EE can improve the inflammatory environment caused by stroke. Compared with MCAO (SE), the MCAO (EE) group reduced the expression of microglia in the ipsilateral hippocampal CA1 and CA3 regions, and decreased serum the levels of pro-inflammatory factors IL-1 $\beta$ and IL-6. However, there was no statistical difference in the myelination and microglia expression level of EE compared with SE in sham group rats, indicating EE don't have effect on white matter of normal rats.

Ischemic stroke is a complex pathophysiological process. It is well known that ischemic stroke can cause gray matter damage. However, stroke can also cause severe white matter damage, which is a risk factor for the high incidence of stroke and poor neurological prognosis. White matter damage has been reported in 64 to $86 \%$ of stroke patients (18). In fact, because white matter receives less collateral circulation than gray matter and has less blood supply, it is usually more severely damaged than gray matter during ischemia (19). Most of the damage caused by stroke is located in the subcortical area. It is worth noting that white matter accounts for nearly half of the average volume of cerebral infarction. Cognitive dysfunction after stroke is one of the clinical symptoms related to white matter damage, which is closely related to the destruction and remodeling of white matter connectivity (20). The pathological changes of white matter damage caused by cerebral ischemia include demyelination, swelling of myelin and destruction of axons of the corpus callosum (21). Our results showed that the MCAO (SE) group had a significant loss of myelin sheath, while the MCAO (EE) group had more complete myelin sheath(Fig. 3). In normal rats, rats in EE group has no obvious changes in myelin sheath(Fig. 3). This indicates that EE can preserve or restore white matter integrity after cerebral ischemia.

The main pathological manifestations of white matter damage caused by ischemic stroke are demyelination and oligodendrocyte death (22). White matter repair includes the occurrence of oligodendrocytes and the myelination of demyelinated axons or new axons, which can help rebuild neuronal connections and axon signal transduction (23). Oligodendrogenesis is the process by which oligodendrocyte precursor cells(OPCs) generate mature oligodendrocytes. Unfortunately, the adult brain's capacity for remyelination is limited, at least in part due to the failure of OPCs to differentiate into mature myelin oligodendrocytes. Although in response to oligodendrocyte damage, OPCs close to the damaged area actively proliferate and migrate to the area around the infarct, and jointly promote the recovery of myelin oligodendrocytes, but few OPCs can differentiate into mature myelin, which leads to poor white matter recovery(24). In order to verify whether the enriched environment can promote the production of oligodendrocytes in the hippocampus of rats after stroke and whether they mature and differentiate into functional myelin. We used western blot and immunofluorescence to detect oligodendrocyte precursor cells(Marker: PDGFR-a, NG2) and myelin markers(MBP), and found that there was no difference in OPCs among the groups(Fig. 4). In the MCAO model group, compared with the SE group, the EE group increased the expression of $\mathrm{MBP}(\mathrm{Fig} .4)$. The results indicate that $\mathrm{EE}$ contributes to the production of functional myelin, but EE has no effect on the expression of oligodendrocyte precursor cells. We speculate that $\mathrm{EE}$ does not promote the formation of OPCs. But it plays a more powerful role in promoting the 
differentiation and maturation of oligodendrocyte precursor cells into mature myelin. The underlying mechanism needs further experimental research.

White matter damage, characterized by axon-glia intact loss and demyelination, is an important cause of cognitive dysfunction after ischemia, and is usually related to microglia activation (25). After ischemic stroke, microglia will be rapidly activated, and the activated microglia can have a dual effect on white matter in a phenotype-dependent manner. In the response of specific microenvironmental signals, proinflammatory M1 microglia dominate about a week after brain injury, and are generally considered to aggravate the cell death and demyelination of oligodendrocytes. One week after brain injury, the newly recruited microglia at the injury site are mainly anti-inflammatory $M 2$, which can relieve local inflammation, promote remyelination, and thus promote white matter repair. It has been reported that M2 microglia become an important part of the regeneration response of demyelinating diseases of the central nervous system by promoting the differentiation of oligodendrocytes (26). Therefore, in order to study the effect of EE on microglia after stroke, we used western blot and immunofluorescence to measure the microglia marker protein IBA-1 in hippocampus, and found that in the MCAO model group, EE significantly reduces the expression of microglia, suggesting that EE may affect the expression of microglia to promote white matter recovery(Fig. 6). However, in the healthy tissues on the contralateral side of the brain injury and the Sham group, the expression of microglia did not show statistical differences, indicating that EE has no effect on the expression of microglia in normal brain tissues, and only affects the microglia in the brain injury area(Fig. 6).

Furthermore, the correlation of cognitive function outcomes with the relative expression of MBP and Iba-1 in ipsilateral hemispheres was analyzed. The cognitive function outcomes positively correlated with the relative expression of MBP and negatively with the relative expression of Iba-1(Fig. 8).

Microglia are the endogenous immune cells of the central nervous system. Cerebral ischemia will cause the activation of microglia. The activated microglia will produce related pro-inflammatory cytokines, such as tumor necrosis factor- $a$ (TNF- $a$ ), interleukin (IL)-1 $\beta$, interferon- $\gamma$ (IFN- $\gamma$ ), IL-6, etc. Most studies have shown that microglia activation leads to the damage of oligodendrocytes/OPCs through the production of pro-inflammatory cytokines. Among them, IL-1b and IL- 6 play an important role in the survival, differentiation and maturation of oligodendrocytes(21) (27). We measure the content of IL-1 $\beta$ and IL- 6 by ELISA, and it was found that the content of the two pro-inflammatory factors was reduced in the MCAO (EE) group compared to the MCAO (SE) group(Fig. 7). Therefore, we speculate that the enriched environment is to reduce the pro-inflammatory factors produced by microglia activation by regulating the expression of microglia, so as to promote the recovery of white matter and the improvement of cognitive function after stroke. The potential specific mechanism of enriched environment affecting microglia needs further research.

\section{Conclusion}


In summary, enriched environment improves cognitive functional recovery possibly by promoting white matter repair. In addition, Our study also demonstrated that exposure to EE effectively suppressed adverse microglia activation/proliferation and microglia-mediated inflammatory factors, preserved white matter integrity after cerebral ischemia-induced white matter injury. By modifying detrimental microglia inflammatory responses implementing EE and even (poly) pharmacological approaches might represent a promising therapeutic strategy for post stroke cognitive impairment.

\section{Abbreviations}

EE: enriched environment

SE: standard environment

MCAO: middle cerebral artery occlusion

MWM: Morris Water Maze

TTC: triphenyltetrazolium chloride

LFB: Luxol-Fast Blue

MBP: myelin basic protein

WMLs: white matter lesions

EAE: experimental autoimmune encephalomyelitis

SD: Sprague Dawley

WM: white matter

OPCs: oligodendrocyte precursor cells

\section{Declarations}

\section{Ethics approval and consent to participate}

The experimental protocol of this study were approved by the Animal Ethical Committee of Shanghai University of Traditional Chinese Medicine.

Consent for publication Not applicable

\section{Availability of data and materials}


The data used to support the findings of this study are available from the corresponding authors upon request. All regents used in this study are available from scientific supply companies.

\section{Competing interests}

The authors of this manuscript have no conflicts of interest to disclose.

\section{Funding}

This study was supported by the Leading Personnel Training Project of Shanghai Pudong New District Municipal Health Bureau in China, No. PWR12018-04 (to XB); the Scientific Research Projects of Shanghai Municipal Health Bureau in China, No. 201940031(to XB).

\section{Authors' contributions}

Yi-Sha Guo performed the experiment and wrote the manuscript. Mei Yuan and Yu Han designed the structure of the manuscript. Xin-Ya Shen and Zhen-Kun Gao managed the literature searches and analyses. Xia Bi assisted with the improvement of the manuscript. All authors contributed to and have approved the final manuscript.

\section{Acknowledgements}

The authors acknowledge the expert contributions of Xia Bi and the assistance of other colleagues.

\section{References}

1. Sun JH, Tan L, Yu JT. Post-stroke cognitive impairment: epidemiology, mechanisms and management. Ann Transl Med. 2014;2(8):80.

2. Pantoni L. Cerebral small vessel disease: from pathogenesis and clinical characteristics to therapeutic challenges. Lancet Neurol. 2010;9(7):689-701.

3. Jokinen H, Kalska H, Ylikoski R, Madureira S, Verdelho A, van der Flier WM, et al. Longitudinal cognitive decline in subcortical ischemic vascular disease-the LADIS Study. Cerebrovasc Dis. 2009;27(4):384-91.

4. Anrather J, ladecola C. Inflammation, Stroke. An Overview Neurotherapeutics. 2016;13(4):661-70.

5. Xu S, Lu J, Shao A, Zhang JH, Zhang J. Glial Cells: Role of the Immune Response in Ischemic Stroke. Front Immunol. 2020;11:294.

6. Perego C, Fumagalli S, De Simoni MG. Temporal pattern of expression and colocalization of microglia/macrophage phenotype markers following brain ischemic injury in mice. J Neuroinflammation. 2011;8:174.

7. Iadecola C, Anrather J. The immunology of stroke: from mechanisms to translation. Nat Med. 2011;17(7):796-808. 
8. Clausen BH, Lambertsen KL, Babcock AA, Holm TH, Dagnaes-Hansen F, Finsen B. Interleukin-1 beta and tumor necrosis factor-alpha are expressed by different subsets of microglia and macrophages after ischemic stroke in mice. J Neuroinflammation. 2008;5:46.

9. Mering S, Jolkkonen J. Proper housing conditions in experimental stroke studies-special emphasis on environmental enrichment. Front Neurosci. 2015;9:106.

10. Forbes T, Goldstein E, Dupree J, Jablonska B, Scafidi J, Adams K, et al. Environmental enrichment ameliorates perinatal brain injury and promotes functional white matter recovery. \#N/A. 2020;11(1):964.

11. Magalon K, Cantarella C, Monti G, Cayre M, Durbec P. Enriched environment promotes adult neural progenitor cell mobilization in mouse demyelination models. Eur J Neurosci. 2007;25(3):761-71.

12. Komitova M, Perfilieva E, Mattsson B, Eriksson P, Johansson B. Enriched environment after focal cortical ischemia enhances the generation of astroglia and NG2 positive polydendrocytes in adult rat neocortex. Exp Neurol. 2006;199(1):113-21.

13. Deng Y, Lu J, Sivakumar V, Ling EA, Kaur C. Amoeboid microglia in the periventricular white matter induce oligodendrocyte damage through expression of proinflammatory cytokines via MAP kinase signaling pathway in hypoxic neonatal rats. Brain Pathol. 2008;18(3):387-400.

14. Jalal FY, Yang Y, Thompson J, Lopez AC, Rosenberg GA. Myelin loss associated with neuroinflammation in hypertensive rats. Stroke. 2012;43(4):1115-22.

15. Vorhees CV, Williams MT. Morris water maze: procedures for assessing spatial and related forms of learning and memory. Nat Protoc. 2006;1(2):848-58.

16. Tsuyama J, Nakamura A, Ooboshi H, Yoshimura A, Shichita T. Pivotal role of innate myeloid cells in cerebral post-ischemic sterile inflammation. Semin Immunopathol. 2018;40(6):523-38.

17. Lalancette-Hébert M, Gowing G, Simard A, Weng YC, Kriz J. Selective ablation of proliferating microglial cells exacerbates ischemic injury in the brain. J Neurosci. 2007;27(10):2596-605.

18. Li L, Simoni M, Küker W, Schulz UG, Christie S, Wilcock GK, et al. Population-based case-control study of white matter changes on brain imaging in transient ischemic attack and ischemic stroke. Stroke. 2013;44(11):3063-70.

19. Wang Y, Liu G, Hong D, Chen F, Ji X, Cao G. White matter injury in ischemic stroke. Prog Neurobiol. 2016;141:45-60.

20. Mifsud G, Zammit C, Muscat R, Di Giovanni G, Valentino M. Oligodendrocyte pathophysiology and treatment strategies in cerebral ischemia. CNS Neurosci Ther. 2014;20(7):603-12.

21. Shigemoto-Mogami Y, Hoshikawa K, Goldman JE, Sekino Y, Sato K. Microglia enhance neurogenesis and oligodendrogenesis in the early postnatal subventricular zone. J Neurosci. 2014;34(6):2231-43.

22. Back SA, Han BH, Luo NL, Chricton CA, Xanthoudakis S, Tam J, et al. Selective vulnerability of late oligodendrocyte progenitors to hypoxia-ischemia. J Neurosci. 2002;22(2):455-63.

23. Zhang R, Chopp M, Zhang ZG. Oligodendrogenesis after cerebral ischemia. Front Cell Neurosci. 2013;7:201. 
24. Liu S, Jin R, Xiao AY, Zhong W, Li G. Inhibition of CD147 improves oligodendrogenesis and promotes white matter integrity and functional recovery in mice after ischemic stroke. \#N/A. 2019;82:13-24.

25. Qin C, Fan WH, Liu Q, Shang K, Murugan M, Wu LJ, et al. Fingolimod Protects Against Ischemic White Matter Damage by Modulating Microglia Toward M2 Polarization via STAT3 Pathway. Stroke. 2017;48(12):3336-46.

26. Jiang X, Pu H, Hu X, Wei Z, Hong D, Zhang W, et al. A Post-stroke Therapeutic Regimen with Omega-3 Polyunsaturated Fatty Acids that Promotes White Matter Integrity and Beneficial Microglial Responses after Cerebral Ischemia. TransI Stroke Res. 2016;7(6):548-61.

27. O'Kusky J, Ye P. Neurodevelopmental effects of insulin-like growth factor signaling. Front Neuroendocrinol. 2012;33(3):230-51.

\section{Figures}

A

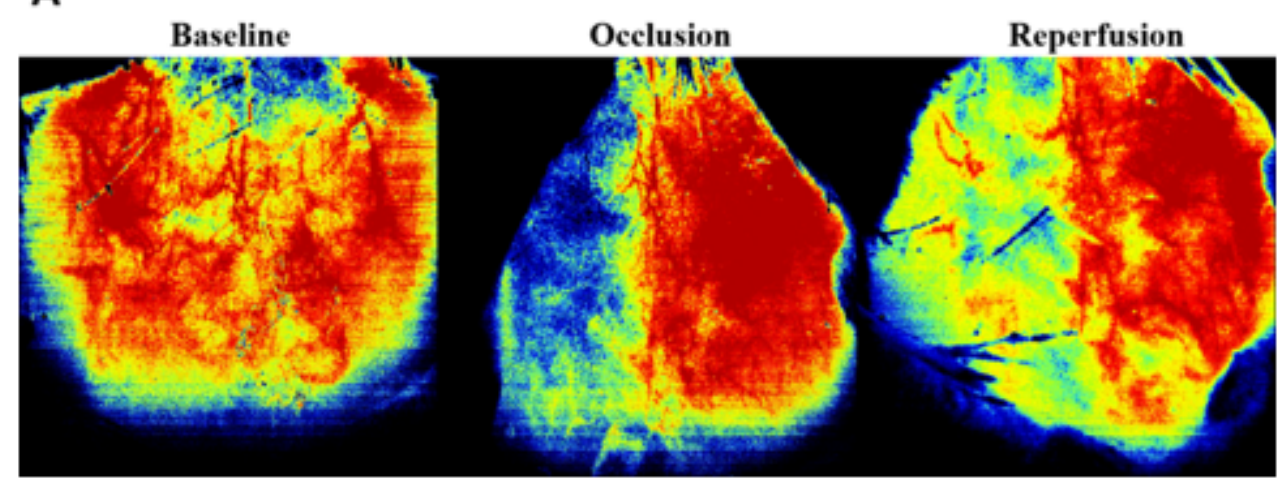

B

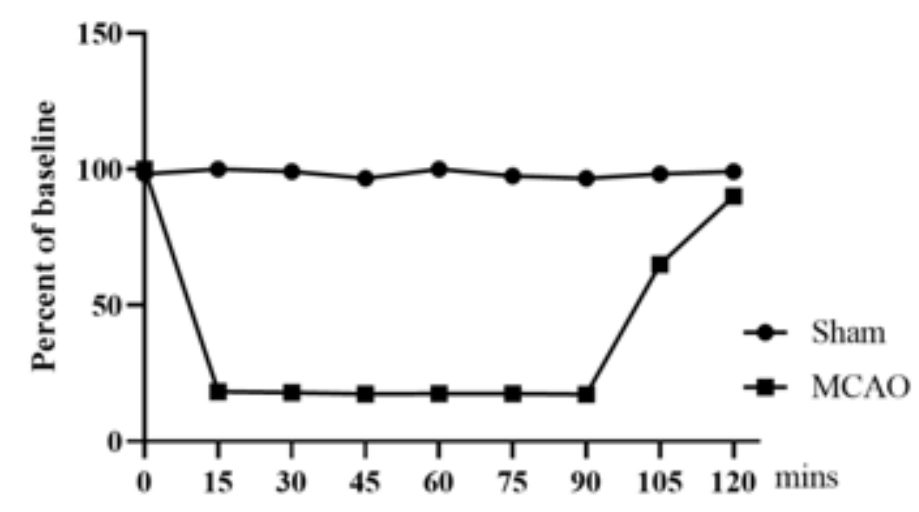

Figure 1

Changes in blood flow in the rat brain during MCAO surgery. (A) An atlas of local blood flow changes in the rat brain generated by laser Doppler flowmetry during MCAO surgery. (B) Quantify of the rCDF. rCDF was mearsured during 120 minutes post-occlusion. 


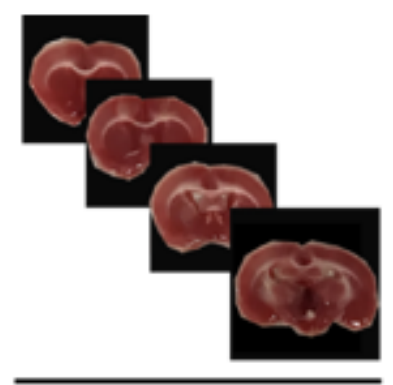

Sham

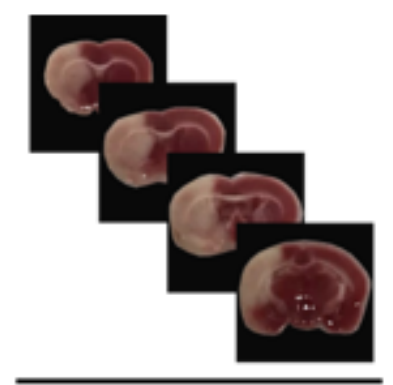

MCAO

\section{Figure 2}

Evaluation of cerebral infarct area using TTC staining. The red area is healthy tissue and the white area is cerebral infarction.

A

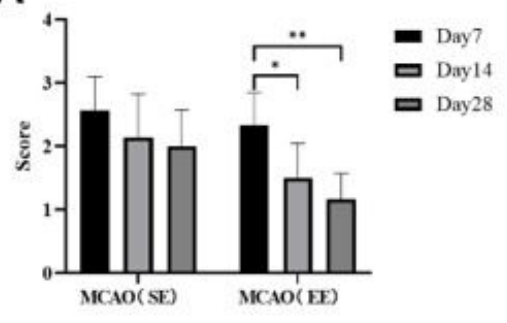

B Learning

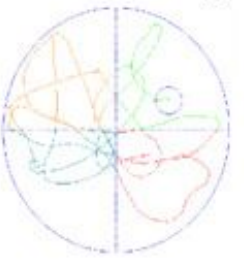

MCAO SE

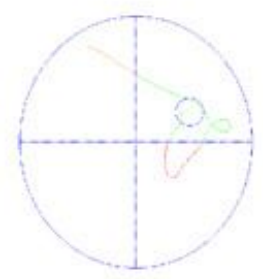

MCAO EE

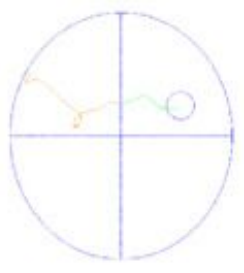

Sham SE

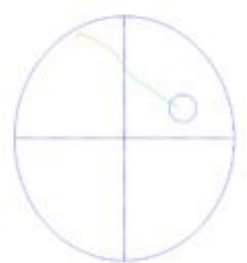

Sham EE

D

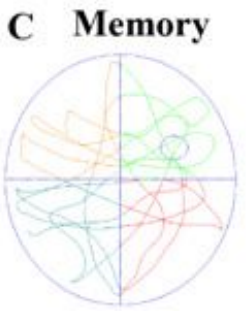

MCAO SE

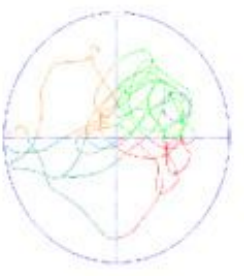

MCAO EE

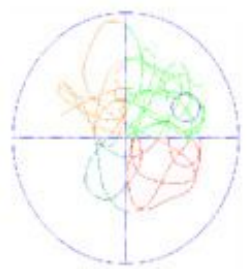

Sham SE

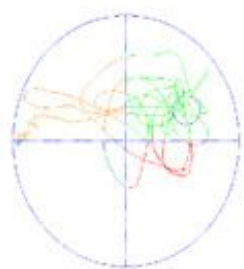

Sham EE

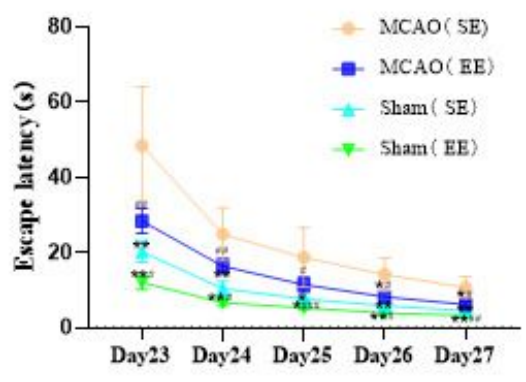

E

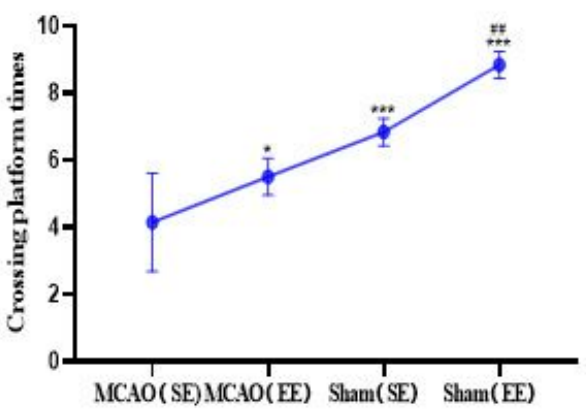

Figure 3 
Effects of enriched environment on neurological impairment and cognitive function in rats.

(A)Neurological scores were assessed on the 7, 14, 28 days in MCAO rats. $n=3 /$ group, ${ }^{*}<0.05,{ }^{\star *} P<0.01$.

Shown are the mean \pm SD. $(B, C)$ Representative images of the swim paths of mice in each group while the platform was present $(B$, the last day of the spatial acquisition trials) and after it was removed $(C$, the probe test), $B$ reflects the spatial learning ability of rats and $C$ reflects the spatial memory ability. (D)The escape latency recorded in the five-day spatial acquisition test; $(E)$ The crossing platform times. $n=$ 3 /group. Shown are the mean $\pm S D$. ${ }^{*}<0.05,{ }^{* \star} P<0.01,{ }^{\star} \star * P<0.01$ vs. $M C A O(S E)$ group; $\# P<0.05, \# \# P<0.01$, $\# \# \# P<0.001$ vs. Sham(SE) group. SE, standard environment; EE, enriched environment.

A

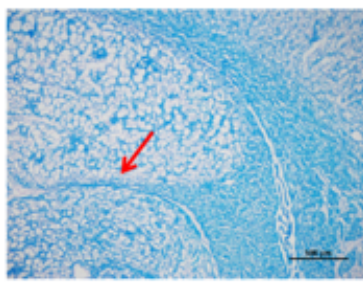

$\mathrm{MCAO}+\mathrm{SE}$

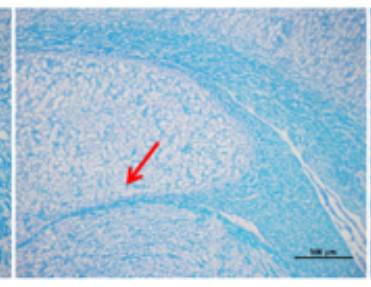

MCAO+EE

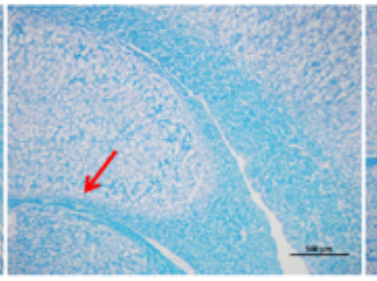

Sham+SE
B

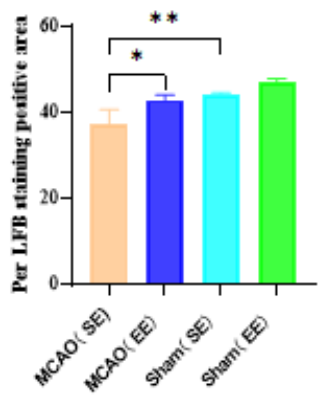

Figure 4

LFB stained sections from hippocampal CA3 in each group. (A)Nerve myelin sheath is blue, and other components are almost colorless. $n=3 /$ group, Scale bar represents $500 \mu \mathrm{m}$. Arrows indicate the difference in recovery of remyelination. (B)Histograms showing the percentage LFB staining positive area. $M C A O+E E$ and Sham+SE showed a higher percentage of the LFB positive staining compared with $M C A O+S E(n=3 /$ group, * $P<0.05$, ** $P<0.01)$. 

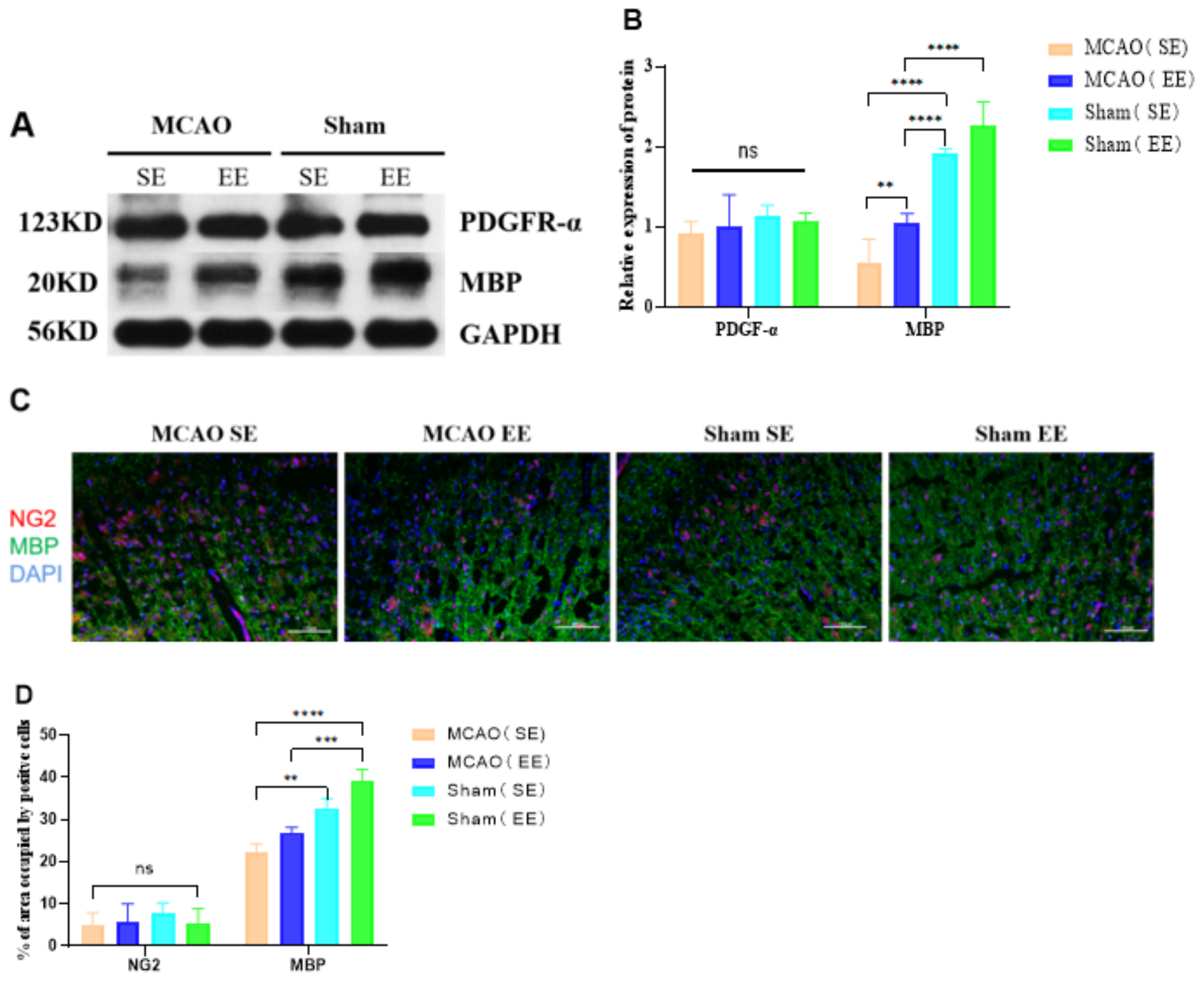

\section{Figure 5}

EE can promote the expression of remyelination-related proteins. (A)Representative western blot for PDGFR-aand MBP in the hippocampus of the four experimental groups. (B)Quantification of PDGFR-aand MBP protein levels, normalized to GAPDH. $n=3$ /group $\varangle \star \star P<0.01$, ${ }^{\star \star \star \star} P<0.0001$. (C) Representative double immunolabeling images of ipsilateral hippocampus CA3 by antibodies directed against NG2 and MBP in each group. Scale bar $=100 \mu \mathrm{m}$. (D)Histograms showing percentage of positive area of NG2 and MBP occupied in overall view in each group. $n=3$ /group, ${ }^{\star \star P}<<0.01,{ }^{\star \star \star} P<0.001$, ${ }^{\star \star \star \star} P<0.0001$. 
A
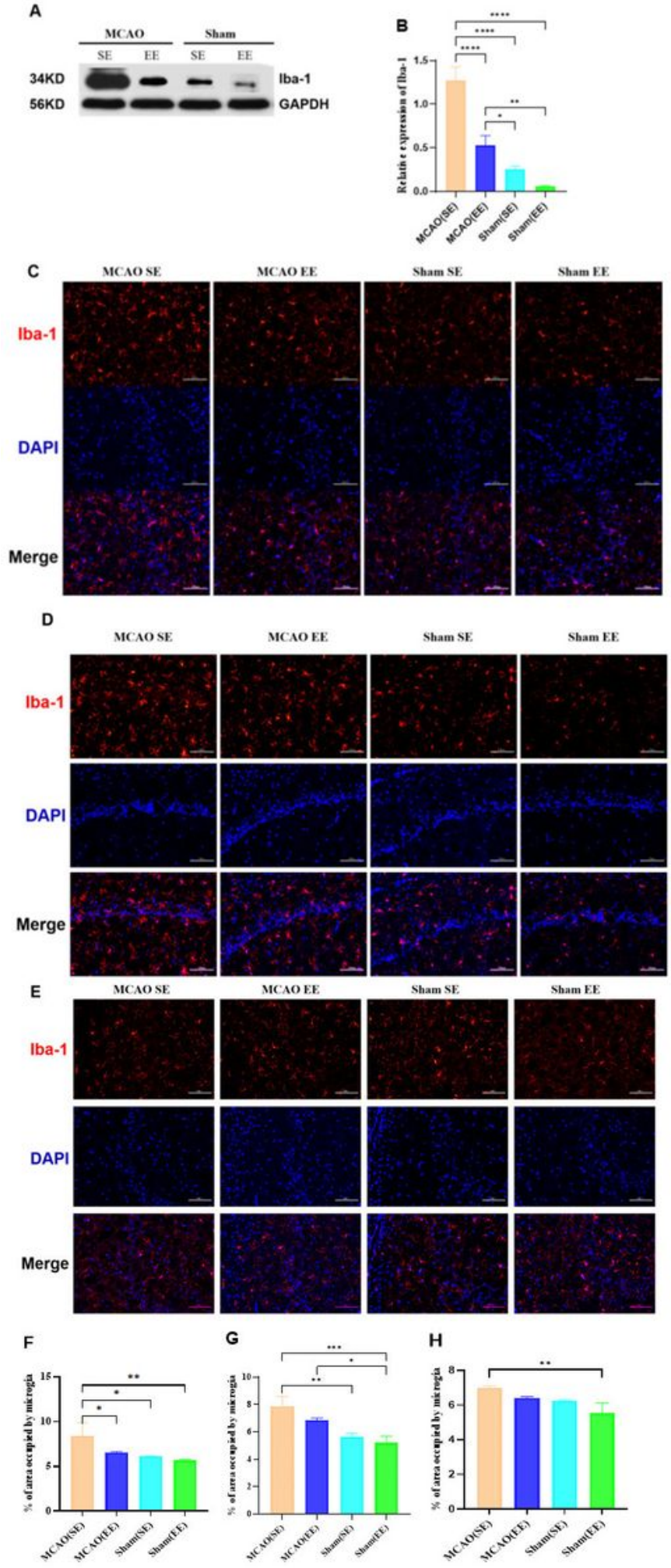

Figure 6

Assessment of microglial proliferation and activation in the hippocampus. (A)Representative western blot for IBA-1 (the marker of microglia) in the hippocampus of the four experimental groups. (B)Quantification of IBA-1 protein levels, normalized to GAPDH. $n=3$ /group ${ }^{*} \mathrm{P}<0.05$, ${ }^{* \star} \mathrm{P}<0.01$, ${ }^{\star * \star *} \mathrm{P}<0.0001$.

(C)Representative immunofluorescence images of hippocampus CA3 of the ipsilateral microglia (red) in each group. Scale bar $=100 \mu \mathrm{m}$. (D)Representative immunofluorescence images of hippocampus CA1 of 
the ipsilateral microglia (red) in each group. Scale bar $=100 \mu \mathrm{m}$. (E)Representative immunofluorescence images of hippocampus CA3 of the contralateral microglia (red) in each group. Scale bar $=100 \mu \mathrm{m}$. $(F \sim H)$ Histograms showing degree of microglial proliferation in each subgroup. $n=3 /$ group $\otimes * P<0.05$, ** $P$ $<0.01, * \star * P<0.001$.

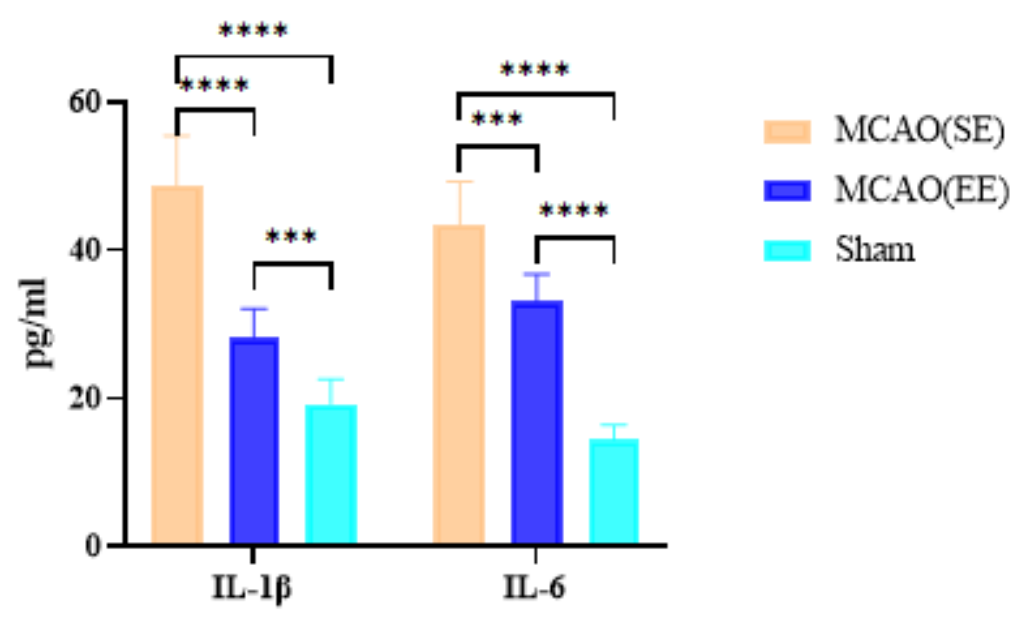

Figure 7

Effect of EE on neuroinflammation in serum of MCAO rats. ELISA showed that EE treatment significantly reduced the levels of serum IL-1 $\beta$ and IL- 6 in MCAO rats. Shown are the mean $\pm S D$. $n=3 /$ group, $\star \star \star * P<0.001,{ }^{\star \star * \star * P}<0.0001$.

A

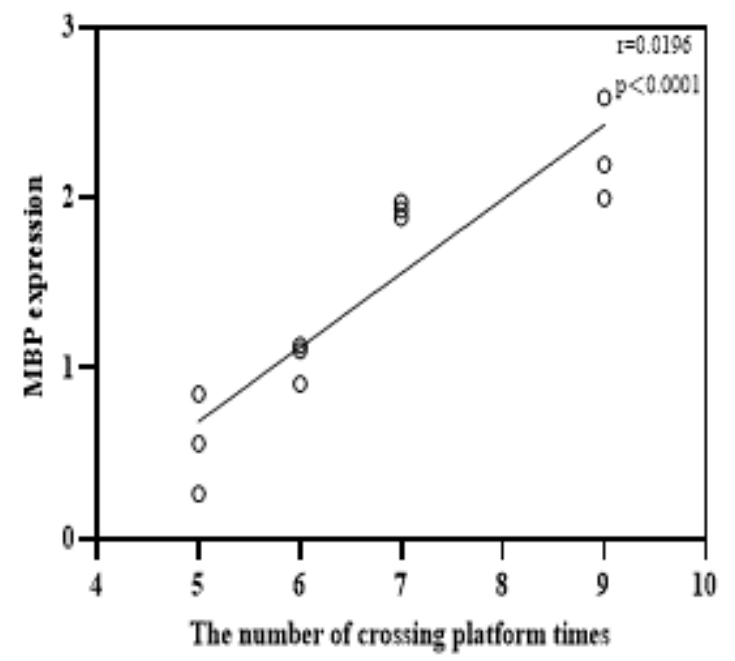

B

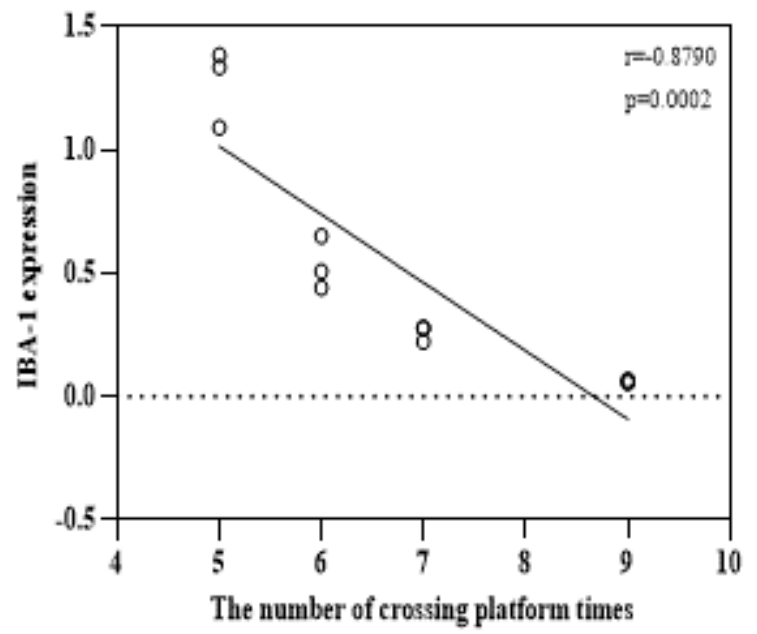

Figure 8

Correlation analysis. (A)Correlation between cognitive function outcomes and MBP expression levels.

(B)Correlation between cognitive function outcomes and IBA-1 expression levels. $\mathrm{P}<0.05$ was considered statistically significant. 
Page 21/21 